\title{
Substantiation of Principles of Construction \\ of Small-Sized Onboard Station of Formation \\ of Anticipatory Hindrances to Laser Control Systems \\ of Fire of an Antiaircraft-Artillery Complex
}

\author{
Yuri L. Koziratsky, \\ Ruslan E. Merkulov and Nikita S. Koch* \\ Military Education and Research Centre of Military-Air Forces \\ "Military-Air Academy \\ Named After Professor N.E. Zhukovsky and Yu.A. Gagarin" \\ 54a Starykh Bolshevikov Str., Voronezh, 394064, Russia
}

Received 30.11.2016, received in revised form 07.02.2017, accepted 19.03.2017

\begin{abstract}
In work researches and possibility of construction of a method of protection of the distantsionnopiloted flying machine from an antiaircraft-artillery complex of the potential opponent, by installation on the given flying machine of small-sized station of the active anticipatory hindrances are resulted, capable to find out pregenerational spontaneous radiation of the transferring channel of the laser range finder which is a part of a laser control system by fire of an antiaircraft-artillery complex and to execute statement to the given means aim imitation a hindrance. The carried out mathematical calculations have shown that the onboard station of hindrances is capable to find out pregeneration spontaneous radiation with probability not more low 0,9 at range to $8 \mathrm{~km}$ and to carry out optical-electronic suppression, systems of a laser range finder, an antiaircraftartillery complex.
\end{abstract}

Keywords: the antiaircraft-artillery complex, the distend piloted flying machine, a laser range finder, station of active anticipatory hindrances.

Citation: Koziratsky Yu.L., Merkulov R.E., Koch N.S. Substantiation of principles of construction of small-sized onboard station of formation of anticipatory hindrances to laser control systems of fire of an antiaircraft-artillery complex, J. Sib. Fed. Univ. Eng. technol., 2018, 11(1), 6-15. DOI: 10.17516/1999-494X-0001.

(c) Siberian Federal University. All rights reserved

* Corresponding author E-mail address: urleo@bk.ru 


\title{
Обоснование принципов построения
}

\section{малогабаритной бортовой станции}

формирования упреждающих помех лазерным системам

\section{управления огнем зенитно-артиллерийского комплекса}

\author{
Ю.Л. Козирацкий, Р.Е. Меркулов, Н.С. Кох \\ Военный учебно-научный цуентр Военно-воздушных сил \\ «Военно-воздушная академия \\ имени профессора Н.Е. Жуковского и Ю.А. Гагарина» \\ Россия, 394064, Воронеж, ул. Старых Большевиков, $54 а$
}

В работе приведень исследования $u$ возможность построения метода зашить дистанционно-пилотируемого летательного аппарата от зенитно-артиллерийского комплекса потенциального противника путем установки на данный летательный аппарат малогабаритной станциии активных упреждающих помех, способной обнаружить предгенерационное спонтанное излучение передающего канала лазерного дальномера, входящего в состав лазерной системы управления огнем зенитно-артиллерийского комплекса и выполнить постановку данному средству прицельную имитационную помеху. Проведеннье математические расчеты показали, что бортовая станция помех способна обнаружить предгенерационное спонтанное излучение с вероятностью не ниже 0,9 на дальности до 8 км и осуществить оптико-электронное подавление системы лазерного дальномера, зенитноартиллерийского комплекса.

Ключевые слова: зенитно-артиллерийский комплекс, дистанционно-пилотируемый летательный аппарат, лазерный дальномер, станцฺия активных упреждающих помех.

\section{Введение}

В настоящее время активно ведутся научно-исследовательские и опытно-конструкторские работы по созданию роботизированных средств вооружения и разработке рациональных форм и способов их боевого применения в войнах и вооруженных конфликтах различного масштаба и интенсивности. Достаточно значимые успехи достигнуты в области создания и применения дистанционно-пилотируемых летательных аппаратов (ДПЛА) в интересах повышения эффективности радиоэлектронной разведки, радиоэлектронной борьбы, поражения стационарных и малоподвижных объектов, радиоэлектронных объектов телекоммуникационной сети, контроля эффективности различных действий.

Бурное использование ДПЛА вызвало во многих странах мира ответную реакцию, направленную на уничтожение таких летательных аппаратов.

Совершенствование систем поражения ДПЛА идет по пути использования комплексированных средств разведки, прицеливания (координатометрии) и управления. Все это помогает противнику преодолевать трудности, связанные с обнаружением ДПЛА, выполненных из композитных материалов, и с использованием радиопоглощающей «обмазки» внутренних металлических элементов конструкции. Как показывает практика, при применении таких лазерных систем управления огнем (ЛСУО) в составе зенитноартиллерийского комплекса (ЗАК) вероятность поражения боеприпасом цели составляет 
порядка $P_{\text {nop }}=0,65 \pm 0,07$. При исключении из состава системы лазерного дальномера величина ошибок в прицеливании увеличивается, а вероятность поражения цели снижается, становится равной $P_{n o p}=0,07$ [1].

Целью данной работы является разработка методического подхода и обоснования малогабаритной бортовой станции формирования упреждающих помех для защиты ДПЛА от огня ЗАК с лазерными системами прицеливания.

\section{Постановка задач}

Подавление оптико-электронных средств связано с преодолением некоторых трудностей: узкополосность поля зрения приёмника ЛД (лазерный дальномер) ЗАК, малая расходимость его зондирующего излучения, небольшая длительность зондирующих импульсов, отсутствие достоверной априорной информации об ориентации лазерного средства. Эти трудности усложняются ещё и тем, что некоторые лазерные средства могут работать в режиме нерегулярной посылки зондирующих импульсов, а также использовать кодирование лазерного излучения, затрудняющее разведку. Поэтому при организации оптико-электронного подавления лазерных средств основные сложности возникают не из-за отсутствия эффективных способов постановки помех, а из-за отсутствия добротных способов информационного обеспечения. Разработка высокоэффективных способов разведки, как показано в [2], может базироваться на основе приема и анализа спонтанных излучений передающего канала лазерного средства. Проведенный анализ результатов работ [3] показал, что в состоящих на вооружении и разрабатываемых лазерных устройствах не предусматривается такая мера помехозащиты, как перекрытие передающего канала на время нарастания импульса индуцированного излучения, что позволяет обеспечить обнаружение предгенерационного спонтанного излучения передающего канала ЛД, а также постановку упреждающих помех до прихода зондирующего излучения лазерного средства противника.

Анализируя современные ЗАК потенциального противника, можно сделать вывод, что наибольшее распространение в данной технике получили импульсные ЛД, в качестве активной среды которых используется алюмо-иттриевый гранат, легированный ионами неодима, излучающий в режиме модулированной добротности [3].

На основании данных сведений требуется определить интенсивность предгенерационного спонтанного излучения.

\section{Определение интенсивности}

\section{предгенерационного спонтанного излучения лазерного дальномера}

Для оценки интенсивности предгенерационного спонтанного излучения ЛД необходимо оценить изменения плотности инверсной населенности во времени в течение импульса накачки. Для лазеров, работающих по четырехуровневой схеме генерации $n_{3}(\mathrm{t}) \approx \Delta(\mathrm{t})$, изменения плотности инверсной населенности во времени можно описывать с помощью дифференциального уравнения

$$
\begin{array}{r}
\frac{d \Delta(t)}{d t}=W_{14} n_{0}-\left(\mathrm{W}_{14}+\bar{W}_{32}\right. \\
\left.+\frac{1}{\tau_{32}}\right) \Delta, \\
-8-
\end{array}
$$


где $\Delta(t)$ - плотность инверсной населенности в единице объема к моменту времени $\mathrm{t} ; W_{14}$ интенсивность накачки (переход активных частиц из состояния 1 в состояние 4 под действием системы оптический накачки); $n_{0}$ - плотность активных частиц; $\bar{W}_{32}$ - среднее значение интенсивности индуцируемых переходов из состояния 3 в состояние 2 под действием усреднённого за интервал времени накачки и по длине оптического резонатора оптического излучения (суммарного); $\tau_{32}$ - среднее время жизни активной частицы в возбужденном состоянии.

Решение уравнения (1) для случая прямоугольного импульса накачки, когда $t \leq \tau_{н}$, (где $\tau_{н}-$ длительность оптического импульса накачки) может быть представлено в виде

$$
\Delta(t)=n_{0} \frac{W_{14}}{W_{14}+\bar{W}_{32}+\frac{1}{\tau_{32}}}\left[1-e^{-\left(W_{14}+\bar{W}_{32}+\frac{1}{\tau_{32}}\right) t}\right] .
$$

Значения параметров, входящих в выражение (2), на основе обобщения данных [4] представлены в табл. 1.

С использование выражения (2) и данных табл. 1 получена зависимость изменения плотности инверсной населенности во времени в течение импульса накачки (рис. 1).

Таблица 1. Выбранные исходные данные для расчета $\Delta(\mathrm{t})$

Table 1. The Chosen initial data for calculation $\Delta(\mathrm{t})$

\begin{tabular}{|c|c|c|c|c|}
\hline Параметр & $W_{14}$ & $\bar{W}_{32}$ & $\tau_{32}$ & $n_{0}$ \\
\hline $\begin{array}{c}\text { Единица } \\
\text { измерения }\end{array}$ & $c^{-1}$ & $c^{-1}$ & $c$ & $c M^{-3}$ \\
\hline Величина & $0,15 \ldots 1,5$ & 0,07 & $2,5 \times 10^{-4}$ & $5 \times 10^{19}$ \\
\hline
\end{tabular}

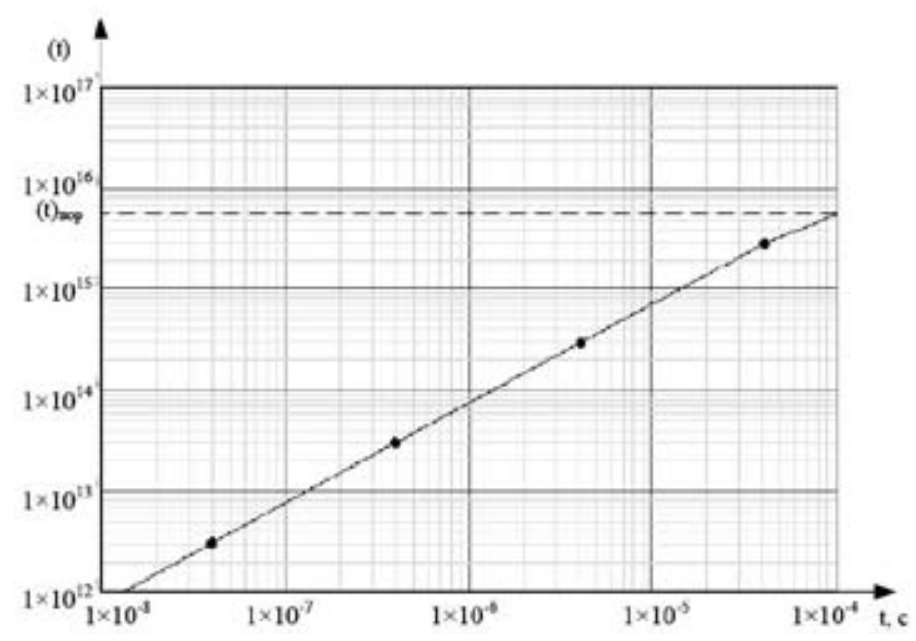

Рис. 1. График изменения плотности инверсной населённости во времени

Fig. 1. The schedule of change of density of inverse density of population in time 
Таблица 2. Выбранные исходные данные для расчета $\bar{S}_{c}$

Table 2. The Chosen initial data for calculation $\bar{S}_{c}$

\begin{tabular}{|l|c|c|c|c|c|c|c|c|}
\hline \multicolumn{1}{|c|}{ Параметр } & $\mathrm{d}$ & $\mathrm{e}$ & $\eta$ & $\tau_{\mu}$ & $\beta$ & 1 & $T_{a}$ & $D_{p}$ \\
\hline Единицы измерения & $\mathrm{мм}$ & $\mathrm{мм}$ & - & $\mathrm{cm}^{2}$ & $\mathcal{M}^{-1}$ & $\mathrm{мм}$ & - & $\mathrm{cm}$ \\
\hline Величина & 5 & 50 & 0,5 & $10^{-8}$ & $2,7 \times 10^{-2}$ & 50 & 0,7 & 5 \\
\hline
\end{tabular}

Таким образом, с помощью уравнения, описывающего изменение плотности инверсной населённости, была определена пороговая плотность инверсной населённости. Теперь необходимо оценить интенсивность спонтанного излучения.

Известно, что не все активные частицы, находящиеся на верхнем энергетическом уровне активного элемента ЛД вследствие так называемой оптической накачки, будут излучены в процессе индуцированного излучения, так как имеет место процесс самопроизвольного перехода активных частиц в состояние с меньшей энергией, называемый спонтанным переходом. Сопровождающее его излучение называется соответственно спонтанным излучением. Поскольку различные активные частицы излучают при этом независимо друг от друга, спонтанное излучение является некогерентным и среднее значение числа сигнальных фотоэлектронов в разведывательном приемнике может быть определено с помощью выражения

$$
\bar{S}_{c}(t)=\frac{A_{32} \pi d^{2} D_{p}^{2} T_{a} \eta \tau_{H} n(t) r_{1}}{64 L^{2}[\sigma \Delta(t)-\beta]} \times\left[e^{[\sigma \Delta(t)-\beta] l}-1\right],
$$

где $A_{32}$ - коэффициент Эйнштейна; $\mathrm{d}$ - диаметр активного элемента; $D_{p}$ - диаметр входной апертуры; $T_{a}$ - потери при распространении излучения через атмосферу; $\eta$ - квантовая эффективность; $\tau_{н}$ - время наблюдения приемника; $\mathrm{n}(\mathrm{t})$ - количество частиц на верхнем энергетическом уровне; $r_{1}$ - коэффициент отражения выходного зеркала резонатора; L - расстояние от выходного торца активного элемента до регистрирующего приёмника; $\sigma$ - поперечное сечение вынужденного перехода рабочих уровней; $\Delta(t)$ - плотность инверсий населённости к моменту времени $\mathrm{t} ; \beta$ - потери в кристалле; 1 - длина активного элемента.

Для нахождения величины сигнальных фотоэлектронов воспользуемся данными [4], приведенными в табл. 2.

На рис. 2 изображен график зависимости величины спонтанного излучения в виде числа фотоэлектронов за время накачки $\left(\tau_{H}\right)$ от дальности $\mathrm{L}=3$ км для различных моментов времени.

На рис. 3 приведены графики зависимостей числа фотоэлектронов от времени для дальности $\mathrm{L}_{1}=1$ км, $\mathrm{L}_{2}=3$ км, $\mathrm{L}_{3}=5$ км, $\mathrm{L}_{4}=10$ км.

Анализируя полученные графики можно сделать вывод, что спонтанное излучение на выходе передающего канала имеет достаточно большую интенсивность в пределах от 1 до 3 км.

\section{Выбор оптимальной схемы приемника}

В качестве оптимального приёмника, установленного на ДПЛА, был рассмотрен приёмник, работающий по правилу Неймана-Пирсона (с квантовым счётчиком в качестве чувстви- 


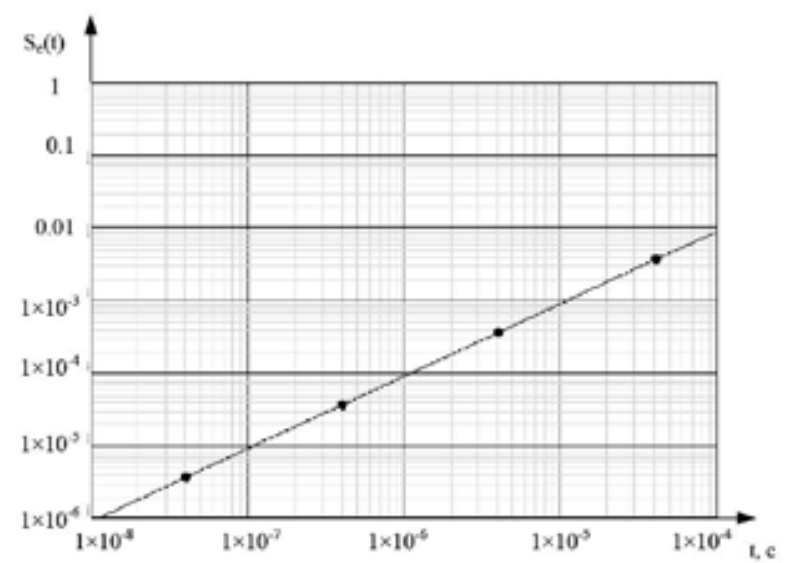

Рис. 2. График зависимости величины интенсивности спонтанного излучения от времени для дальности 3 км

Fig. 2. The schedule of dependence of size of intensity of spontaneous radiation from time for range of $3 \mathrm{~km}$

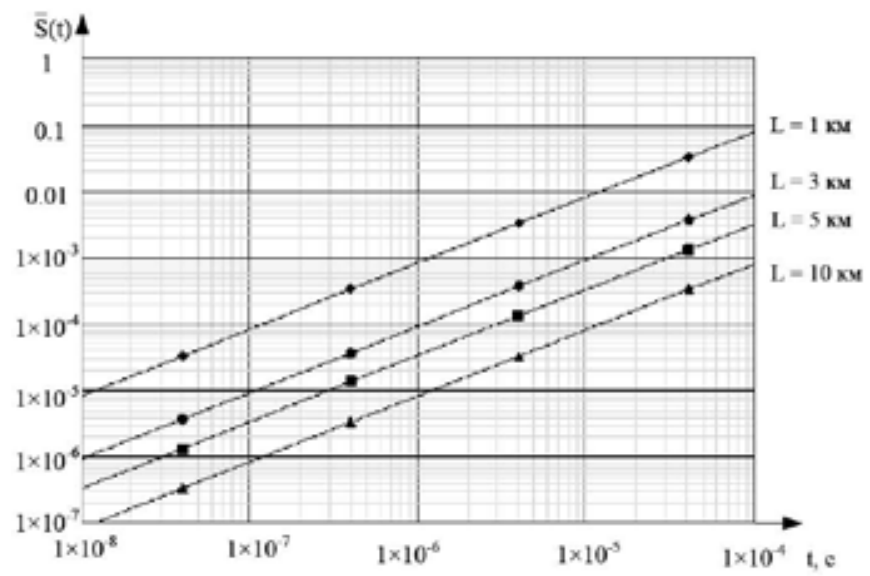

Рис. 3. График зависимости величины интенсивности спонтанного излучения от времени для различных дальностей: $\mathrm{L}_{1}=1$ км, $\mathrm{L}_{2}=3$ км, $\mathrm{L}_{3}=5$ км, $\mathrm{L}_{4}=10$ км

Fig. 3. The schedule of dependence of size of intensity of spontaneous radiation from time for various ranges: $\mathrm{L}_{1}=1 \mathrm{~km}, \mathrm{~L}_{2}=3 \mathrm{~km}, \mathrm{~L}_{3}=5 \mathrm{~km}, \mathrm{~L}_{4}=10 \mathrm{~km}$

тельного элемента). Такого типа приёмник наиболее целесообразно использовать именно при решении задач обнаружения, когда неизвестны априорные вероятности посылки сигналов и цены ошибок при приёме [5].

Критерий Неймана-Пирсона обеспечивает максимум вероятности обнаружения сигнала данной интенсивности при фиксированной вероятности ложного обнаружения [6]. При использовании этого критерия, имеющего на входе приёмника выборку из реализации, $\mathrm{n}_{1}, \mathrm{n}_{2}, \ldots . \mathrm{n}_{N}$ отожествляется с наличием сигнала в том случае, если выполняется неравенство

$$
\begin{gathered}
\lambda\left(\mathrm{n}_{1}, n_{2}, \ldots \mathrm{n}_{N}\right)=\frac{P_{1}\left(\mathrm{n}_{1}, n_{2}, \ldots \mathrm{n}_{N}\right)}{P_{0}\left(\mathrm{n}_{1}, n_{2}, \ldots \mathrm{n}_{N}\right)} \geq \lambda, \\
-11-
\end{gathered}
$$


или отождествляется с наличием шума, если $\lambda<\lambda_{0}$, где $\mathrm{P}_{1}-$ совместная вероятность для совокупности выборочных значений $\mathrm{n}_{1}, \mathrm{n}_{2}, \ldots \mathrm{n}_{N}$ при условии, что сигнал присутствует; $\mathrm{P}_{0}-$ совместная вероятность для той же совокупности выборочных значений при условии, что сигнала нет. Отношение правдоподобия при обнаружении пуассоновского сигнала в пуассоновском шуме

$$
\lambda_{1}=\prod_{i=1}^{N}\left(\frac{\bar{S}_{c i}}{\bar{S}_{u}}+1\right)^{n_{i}} e^{-\bar{S}_{c i}}
$$

где $\bar{S}_{c i}$ - среднее число сигнальных фотоэлектронов (образования переходов) за время накопления; $\bar{S}_{u}-$ среднее число шумовых (помеховых) фотоэлектронов (образования переходов) за время накопления; $N=E\left\{\frac{T}{\tau}\right\}$ - целочисленное значение отношения, $E-$ энергия, Т - время наблюдения, $\tau$ - время накопления приемника.

Решение о наличии полезного сигнала будет приниматься при условии, что $\lambda_{1} \geq 1$ в противном случае принимается решение об отсутствии полезного сигнала [6]. Логарифмируя (5) с учетом изложенного правила принятия решения, находим, что при выполнении условия

$$
\sum_{i=1}^{N} n_{i}\left(\bar{S}_{c i}+1\right) \geq \sum_{i=1}^{N} \bar{S}_{c i}
$$

принимается решение, что принятое колебание является полезным сигналом, в противном случае на вход воздействует шумовая составляющая.

Используя (6), структурную схему приемника (блок обработки сигналов) можно представить таким образом (рис. 4).

Вероятность обнаружения предгенерационного спонтанного излучения может быть оценена из следующего равенства:

$$
P_{\text {обн }}=\phi\left\{\sqrt{\frac{S N \bar{S}_{c}}{1+S}}-\frac{1}{\sqrt{1+S}} \phi^{-1}[F]\right\},
$$

где $\phi(t)=\frac{1}{\sqrt{2 \pi}} \int_{-\infty}^{t} e^{-\frac{x^{2}}{2}} d x-$ интеграл вероятности; $\bar{S}_{c i}=\bar{S}_{c} ; S=\frac{\bar{S}_{c}}{\bar{S}_{u}} ; \phi^{-1}[\phi(\mathrm{x})]=x[4]$.

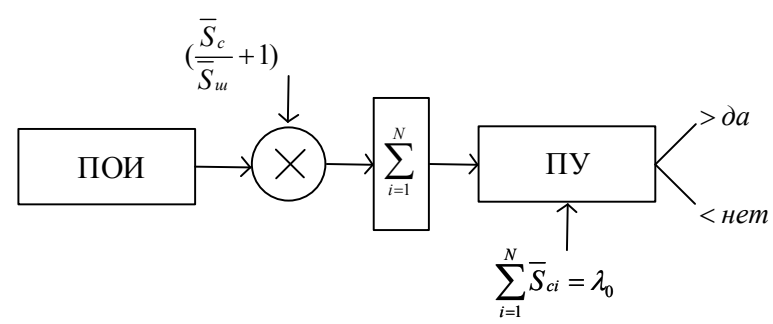

Рис. 4. Оптимальная схема приёмника

Fig. 4. The optimum scheme of the receiver 
Используя зависимость интенсивности спонтанного излучения от времени, для случая, когда накопление спонтанного излучения начинается с момента времени $t_{t}=10^{-5} \mathrm{c}$ и завершается в момент времени $t_{\kappa}=5 \times 10^{-5} c\left(\mathrm{~T}=4 \tau=t_{\kappa}-t_{н}=4 \times 10^{-5} c\right)$, получены графики зависимости вероятности обнаружения предгенерационного спонтанного излучения (рис. 5). Для дальностей 3, 5, 10 км осуществляется наиболее уверенный приём предгенерационного спонтанного излучения с вероятностями обнаружения, $\left(P_{\text {обн }}\right)$, равными 0,8 ; 0,$9 ; \sim 1$.

\section{Выбор схемы устройства создания помех}

Общая структурная схема устройства создания активных упреждающих помех изображена на рис. 6. Средство формирования помех представляет собой некогерентный источник излучения - полупроводниковый инжекционный лазер (линейка полупроводниковых лазеров, включенных параллельно), излучающий на длине волны $\lambda=1,06$ мкм, со временем развития разряда $10^{-9}-10^{-6}$ с. Мощность на выходе лазера аддитивна и может составлять величину до $1,5-2$ МВт [7].

Вероятность оптико-электронного подавления лазерного дальномера СУО ЗАК оценим как произведение вероятности обнаружения спонтанного излучения на вероятность срабатывания лазерного дальномера по помеховому (имитационному) импульсу, сформированному бортовой станцией помех:

$$
P_{\text {оэn }}=P_{\text {обн }} \times P_{c p} .
$$

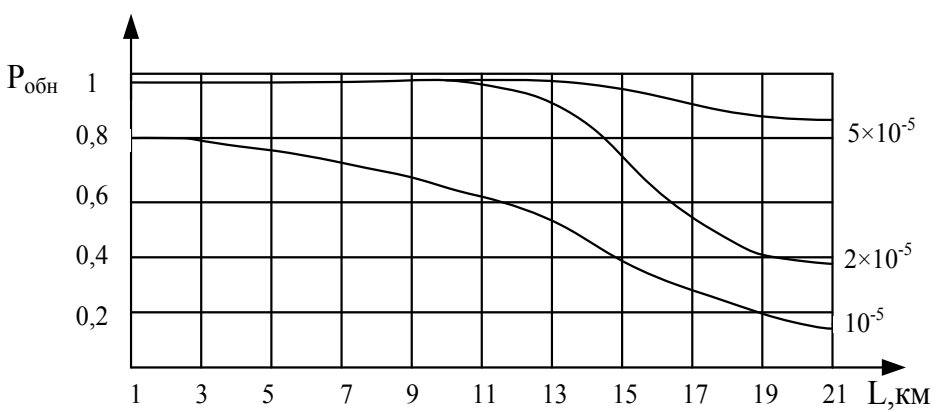

Рис. 5. График зависимости вероятности обнаружения при различных значениях времени

Fig. 5. The schedule of dependence of probability of detection at various values of time

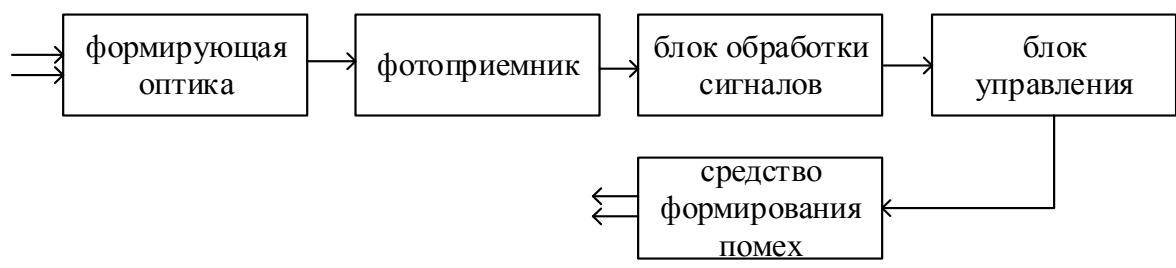

Рис. 6. Структурная схема устройства создания активных упреждающих помех

Fig. 6. The Block diagramme of the device of creation of active anticipatory hindrances 


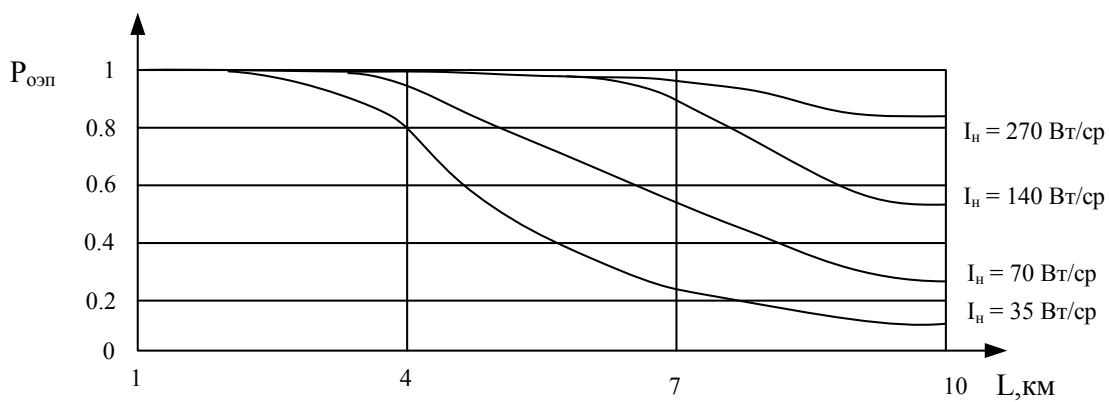

Рис. 7. Графики зависимости вероятности оптико-электронного подавления от дальности при различных значениях интенсивности спонтанного излучения

Fig. 7. Schedules of dependence of probability of optiko-electronic suppression from range at various values of intensity of spontaneous radiation

Вероятность $\mathrm{P}_{\mathrm{cp}}$ оценивается с использованием выражения (7), в котором в качестве полезного сигнала применяется помеховое (имитационное) излучение.

На рис. 7 приведены графики зависимостей вероятности оптико-электронного подавления лазерного дальномера от дальности $\mathrm{L}$ для различных интенсивностей помехового излучения в спектре чувствительности приёмного канала.

Из анализа графиков следует, что при интенсивностях помехового излучения в спектре чувствительности приёмного устройства $270,140,70$ и 35 Вт/ср вероятность срабатывания лазерного дальномера близка к единице на дистанциях 8, 5, 3 и 1 км соответственно.

Анализируя полученные результаты, делаем вывод, что устройство создания активных упреждающих помех можно будет уверенно обнаружить с вероятностью $P_{\text {оби }}=0,9 \div 1$ и эффективно подавлять на расстоянии до 8 км с вероятностью срабатывания лазерного дальномера $P_{c}=0,7 \div 1$ при интенсивностях помехового излучения $I_{n}=140 \div 270 \mathrm{Bm} / \mathrm{cp}$.

Известно, что зенитно-артиллерийские комплексы противника, в состав которых входят лазерные системы дальнометрирования, ведут стрельбу на расстояние 5-8 км. Исходя из анализа данных комплексов, а также характеристик предложенного устройства создания упреждающих помех, можно сделать вывод, что вероятность нормального функционирования ЛСУО ЗАК в условиях постановки имитационных помех ДПЛА составит порядка $P_{\phi}=0,15 \pm 0,07$.

\section{Заключение}

Таким образом, проведённые исследования позволяют сделать вывод о возможности разведки работы ЛСУО по спонтанному излучению, предшествующему индуцированному излучению. Расчёты показали, что интервал времени от момента регистрации спонтанного излучения до момента начала индуцированного излучения порядка $10^{-4}-10^{-3} \mathrm{c}$, что вполне достаточно для создания маскирующих и имитирующих помех, упреждающих основное излучение лазерных средств. Это открывает принципиально новые возможности для эффективной защиты ДПЛА, выполняющего боевую задачу в условиях применения противником ЗАК. 


\section{Список литературы}

[1] Макаров С.А., Корнеев С.В. Военные акции США и Великобритании против Ирака. Журнал МО РФ. Зарубежное военное обозрение, 1999, 6, 23-33 [Makarov S.A., Korneev S.V. Military action the US and UK against Iraq. The journal of the defense Ministry. Foreign military review, 1999, 6, 23-33 (in Russian)]

[2] Козирацкий Ю.Л. Обнаружение и координатометрии оптико-электронных средств, оценка их параметров. М.: Радиотехника, 2015. 456 c. [Kozirazki Y.L. Detection and coordinatefree opto-electronic means, estimation of their parameters. Moscow, Radio Engineering, 2015. 456 p. (in Russian)]

[3] Борисов Е.Г., Евдокимов В.И. Высокоточное оружие и борьба с ним. М.: Лань, 2013. 496 c. [Borisov E.G., Evdokimov V.I. Precision weapons and combating. Moscow, DOE, 2013. 496 p. (in Russian)]

[4] Зверев Г.М., Голлев Ю.Д., Шалаев Е.А., Исакин А.А. Лазеры на алюмоиттриевом гранате с неодимом. М.: Радио и связь, 1985. 144 с. [Zverev G.M., Golev Y.D., Shalaev E.A., Isakin A.A. Lasers alyumoittrievy a neodymium garnet. Moscow, Radio and communication, 1985. 144 p. (in Russian)]

[5] Козирацкий Ю.Л. Модели информационного конфликта средств поиска и обнаружения. М.: Радиотехника, 2013. 232 c. [Kozirazki Y.L. models of information conflict of means of search and discovery. Moscow, Radio Engineering, 2013. 232 p. (in Russian)]

[6] Тихонов В.И. Оптимальный прием сигналов. М.: Радио и связь, 1983. 320 с. [Tikhonov V.I. Optimal signal reception. Moscow, Radio and communication, 1983. 320 p. (in Russian)]

[7] Микаэлян А.Л., Тер-Микаэлян М.Л., Турков Ю.Г. Оптические генераторы на твердом теле. М.: Сов радио, 1967. 384 с. [Mikaelian A.L., Ter-Mikaelyan M.L., Turkov Y.G. Optical generators of the solid. Moscow, Soviet radio, 1967. 384 p. (in Russian)] 